\section{AB010. Management of complications after esophagectomy}

\section{Andrew C. Chang}

Section of Thoracic Surgery, Michigan Medicine, Ann Arbor, MI, USA

Correspondence to: Andrew C. Chang. Section of Thoracic Surgery, Michigan Medicine, TC2120/5344, 1500 East Medical Center Drive, Ann Arbor, MI 48109, USA. Email: andrwchg@umich.edu.

\begin{abstract}
Esophagectomy remains a mainstay in the treatment of esophageal and esophagogastric junction carcinoma. Although achieving adequate oncologic resection is paramount, equally important is the need to restore comfortable swallowing and to maximize quality of life. A wide array of surgical approaches are available and address factors including choice of conduit, location of the primary esophagoenteric anastomosis, and other considerations, particularly inclusion of a gastric emptying procedure and placement of alternative nutritional access. Regardless of approach, complications following esophagectomy occur frequently, with a recent report from a multi-institutional study indicating overall rates of over $50 \%$ patients experiencing at least one complication.
\end{abstract} The occurrence of complications has been associated with worse postoperative mortality, prolonged hospitalization, increased costs of care and worse overall and cancer-free survival. Although mortality following esophagectomy can vary widely across centers and among surgeons, there is less variation observed in rates of complications. Programs with lower operative mortality may have greater collective clinical experience and systems that can recognize and address complications directly, reducing "failure to rescue" at such programs. Pulmonary complications occur most frequently, in over $25 \%$ of patients, consisting of mostly pneumonia, new pleural effusion and respiratory failure. Gastrointestinal complications occur next in frequency, notably esophagoenteric anastomotic leak and delayed conduit emptying. Chylothorax and recurrent laryngeal nerve injury occur less frequently, in $4-5 \%$ of patients. Atrial dysrhythmia requiring intervention, pharmacologic or otherwise, is also observed in 10-20\% of patients and may be a hallmark of an ongoing localized inflammatory response arising from a conduit-related complication. "Enhanced Recovery After Surgery" (ERAS)-protocols capture many elements of perioperative care that have been shown to reduce pulmonary complications. Preoperative regimens that include exercise and chest physiotherapy, intraoperative strategies that address protective lung ventilation and goaldirected fluid management, and postoperative analgesic control are significant features of ERAS that can mitigate pulmonary or non-technical complications. Identification and treatment of recurrent laryngeal nerve injury can reduce aspiration-related pulmonary complications. Protocolbased management of other non-technical complications, such as atrial dysrhythmia, can streamline care although efforts also should continue to identify other complications such as anastomotic leak that might contribute to mediastinal sepsis as a trigger for atrial fibrillation. As an esophageal substitute, the gastric interposition is the conduit of choice for most esophageal surgeons although colon and jejunum remain good options. The incidence of complications at the esophagogastric anastomosis can vary widely and is related greatly to the anastomotic location, with cervical esophagogastric anastomoses having higher reported leak rates compared with intrathoracic placement. Patient and disease-related factors, such as prior history of current smoking history, chronic obstructive pulmonary disease, peripheral vascular disease, diabetes, and particularly preoperative radiation therapy with or without chemotherapy, are risk factors for anastomotic leak. Awareness of these factors should increase vigilance for anastomotic complications. Intraoperative assessment of conduit perfusion can identify whether a conduit has compromised vascular supply that might affect adequate anastomotic healing. Diagnosis of anastomotic leak can be established by contrast or CT esophagogram, endoscopy or clinical suspicion. Measures to avoid mediastinal sepsis can reduce morbid consequences, such as anastomotic stricture, or life-threatening sequelae, particularly the development of tracheo-enteric fistula. For patients with a cervical anastomosis, confirmation of a suspected significant leak is accomplished and managed by immediate exploration with cervical drainage as needed. Management of an intrathoracic anastomotic leak can include stent placement, adequate drainage and endoscopic vacuumassisted closure. If an anastomotic leak is found to be due to conduit necrosis, anastomotic revision with resection of the necrotic portion of conduit or diversion with placement of an end cervical esophagostomy and completion gastrectomy should be considered. Adequate nutrition can be maintained 
by initiation of enteral feeding via a jejunostomy tube, often placed at the time of initial resection. While initial management of low to intermediate-volume chylothorax can include pharmacologic treatment or initiation of noor low-fat enteral nutrition, high-volume chylothorax is a morbid complication that can lead to severe malnutrition, fluid and electrolyte derangement and immunosuppression. The management of chylothorax has changed considerably with increased utilization of interventional radiology. Early lymphangiography and thoracic duct embolization, where such technical expertise is available, can limit the need to return the patient to operation for thoracic duct ligation. Should reoperation be necessary, if the actual site of chylous drainage cannot be identified and ligated directly, mass ligation of the thoracic duct can be achieved via right thoracoscopy or thoracotomy, regardless of the side of chylothorax presentation. Conduit-related complications related to mechanical obstruction can be limited by proper positioning of the intrathoracic conduit with reduction of any redundant conduit back into the abdomen in order to avoid gastric torsion or conduit tortuosity. The diaphragmatic crura should be reapproximated without compromising the vascular supply to the conduit in order to avoid thoracic herniation of viscera, especially the transverse colon. Delayed conduit emptying arises as a result of several technical reasons. Conduit redundancy, particularly when the esophagus has been mobilized through the right chest, can result in tortuosity or even gastric torsion. In preparing the conduit, a wider tubularized stomach might be more prone to tortuosity. Delayed emptying also can arise following an inadequate or absent gastric drainage procedure. Although pyloromyotomy or pyloroplasty at the time of esophagectomy is our preferred approach, the use of botulinum toxin injection at the pylorus has been described particularly for patients undergoing esophagectomy using minimal access techniques. As expertise and experience in "third space" endoscopy develops, gastric peroral pyloroplasty may be considered for patients with delayed conduit emptying. In summary, although complications following esophagectomy occur frequently, early recognition and initiation of treatment have the aim of mitigating longterm morbidity and reducing the mortality that is associated with failure to rescue. Preoperative teaching and physical preparation, intraoperative decision-making and operative technique all have impact on mortality, complications and patient satisfaction following esophagectomy.

Keywords: Esophagectomy; complications; enhanced recovery after surgery; failure to rescue

doi: $10.21037 /$ shc.2019.AB010

Cite this abstract as: Chang AC. Management of complications after esophagectomy. Shanghai Chest 2019;3:AB010. 